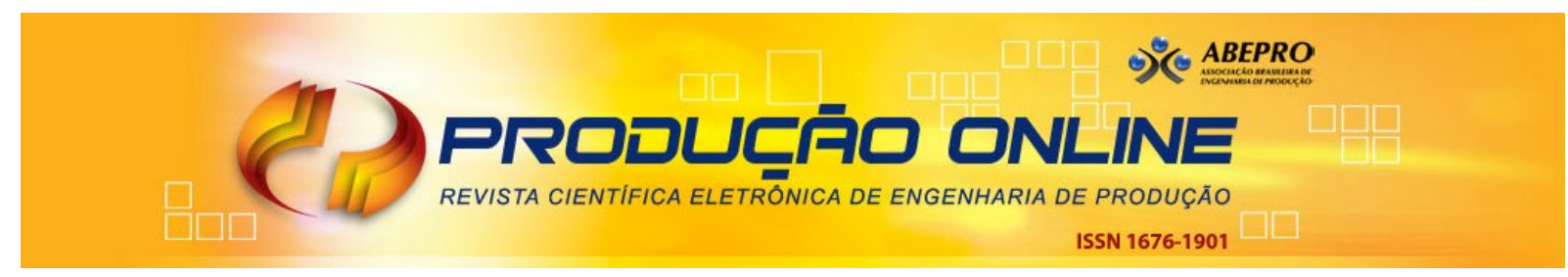

\title{
PROBLEMAS SUPORTADOS PELOS PILOTOS AGRÍCOLAS E SUAS RELAÇÕES COM SEGURANÇA E SAÚDE OCUPACIONAL
}

\section{PROBLEMS INCURRED BY AGRICULTURAL PILOTS AND ITS RELATIONS WITH OCCUPATIONAL HEALTH AND SAFETY}

\author{
Mateus Zanatta* E-mail: mzanatta@producao.ufrgs.br \\ Fernando Gonçalves Amaral** E-mail: amaral@producao.ufrgs.br \\ *Universidade Federal do Rio Grande do Sul (UFRGS), Porto Alegre, RS
}

\begin{abstract}
Resumo: Este estudo faz um levantamento da informação qualitativa dos estudos que abordam os problemas suportados pelos pilotos agrícolas na execução de seu trabalho, que podem estar relacionados à saúde e segurança do trabalho. O objetivo é investigar se a evolução das tecnologias utilizadas na aviação agrícola é acompanhada de uma melhoria das condições de trabalho. Para isto, foi realizada uma revisão sistemática da literatura procurando, através de mecanismos de busca informatizados, reunir a informação existente sobre este tema. A síntese dos dados incluiu os fatores de exposição apresentados e as respectivas relações com segurança do trabalho e saúde ocupacional. Os principais problemas associados à saúde e segurança do trabalho abordados nos estudos incluídos a exposição aos químicos aplicados, a exposição ao ruído e as condições térmicas desfavoráveis, o uso inadequado ou a falta de equipamentos de proteção individual e coletivo, a gestão da equipe de trabalho e a gestão da saúde do trabalho. Apesar de não serem tratados de forma mais aprofundada, outros problemas são apresentados em estudos que avaliaram as condições do ambiente de trabalho. Estes incluem: Capacitação e treinamento, gestão de tempo de trabalho, choques, vibrações, forças gravitacionais, relacionamento pessoal, condições meteorológicas, e responsabilidades do piloto.
\end{abstract}

Palavras-chave: Fatores humanos. Aviação agrícola. Revisão sistemática.

\begin{abstract}
This study assesses qualitative information from studies that address the problems incurred by agricultural pilots in the execution of their work, which may be related to the occupational health and safety. The aim is to investigate whether the evolution of the technologies used in agricultural aviation is accompanied by an improvement in working conditions. In order to achieve this, it was performed a systematic review through computerized search engines to gather existing information on this subject. Data synthesis included the exposure factors presented in work environment and their relations with occupational health and safety. The main problems associated with the health and safety covered in the studies includes exposure to chemicals, noise exposure and unfavorable thermal conditions, improper use or lack of personal and collective protective equipment, team management and occupational health management. Although they are not treated in more detail, other problems are presented in studies that evaluated the conditions of the work environment. These include training and education, working time management, shocks and vibrations, gravitational forces, personal relationships, weather conditions and pilot's responsibilities.
\end{abstract}

Keywords: Human factors. Agricultural aviation. Systematic review.

\section{INTRODUÇÃO}

Revista Produção Online, Florianópolis, SC, v.15, n. 4, p. 1195-1223, out./dez. 2015. 
A aviação agrícola, por vezes referida como operações aeroagrícolas ou aplicação aérea, é apresentada nas regulamentações do setor como uma atividade econômica de aplicação de qualquer substância destinada à nutrição de plantas, tratamento do solo, propagação da vida vegetal, controle de pragas (FAR PART 137, 1965; HRBA 137, 1999). Sendo assim, é uma atividade importante que afeta diretamente a preservação da agricultura, horticultura e floresta. Aproximadamente 1.720 aeronaves para atuação em operações agrícolas estão registradas no Brasil, e aproximadamente 10.000 nos Estados Unidos (GUIMARÃES, 2012). Estas são responsáveis por grande parte dos serviços utilizados para produção agrícola desses países, como por exemplo, a pulverização de lavouras e aplicação de adubo.

No contexto da aviação agrícola observa-se a ocorrência de acidentes e fatalidades, sendo a maior em índice de acidentes na Austrália, com mais de 12 acidentes para cada 100.000 horas de voo entre os anos de 2001 e 2009 (ATSB, 2011), e superior à média de toda a aviação geral nos Estados Unidos, com aproximadamente 5 acidentes para cada 100.000 horas de voo neste mesmo período (NTSB, 2011). Colisão com obstáculos, perda de potência ou falha de motor ou sistema, perda de controle da aeronave e pouso forçado são as principais causas apontadas para os acidentes (ATSB, 2011; CENIPA, 2011; NTSB, 2011). Estes, facilitados pelas condições de aplicação aérea, ou pelos 'fatores de exposição', que para o entendimento deste estudo, representam problemas suportados pelos pilotos agrícolas na execução de seu trabalho, que podem ser resolvidos através de intervenções ergonômicas.

Estudos anteriores que abordaram problemas relacionados ao trabalho consideraram principalmente as situações de alto perigo, como voos efetuados a baixas altitudes (NTSB, 2011), terrenos com vários tipos de obstáculos, como cercas, redes de energia e vegetação (ATSB, 2011; CENIPA, 2011; NTSB, 2011). Esses estudos demonstram a relação entre estes problemas com a ocorrência de acidentes, porém, outros fatores de exposição, presentes em estudos da indústria, aviação civil e militar, não são abordados de forma tão importante na aviação agrícola. Para citar alguns, aceleração gravitacional (AYDOG et al., 2004), vibração (AYDOG et al., 2004; BALASUBRAMANIAN, DUTT \& RAI, 2011; KÅSIN, MANSFIELD \& WAGSTAFF, 2011), ruído (FITZPATRICK, 1988; KÜPPER et al., 
2010; KURONEN et al., 2004), que são apresentados como contribuintes para debilitação da saúde, e em certas situações, estes problemas podem estar comprometendo o desempenho do piloto ao nível de colaborar com a ocorrência de acidentes. Esses fatores de exposição são evidentes em situações de trabalho específicas, sendo necessário maior conhecimento do ambiente de trabalho na aviação agrícola para poder relacioná-los com a Saúde e Segurança do Trabalho (SST).

Para compreender melhor o ambiente de trabalho dos pilotos agrícolas, este estudo faz um levantamento da informação qualitativa dos estudos sobre a aviação agrícola, verificando a existência de abordagens sobre fatores de exposição (também nomeados problemas relacionados ao trabalho), que podem estar relacionados à SST. O objetivo é investigar se a evolução das tecnologias utilizadas na aviação agrícola é acompanhada de uma melhoria das condições de trabalho. Para tal, foi realizada uma revisão sistemática da literatura procurando, através de mecanismos de busca informatizados, reunir a informação existente sobre este tema.

\section{PROCEDIMENTOS METODOLÓGICOS}

Este estudo foi conduzido através de uma revisão sistemática da literatura de acordo com as definições de WRIGHT et al. (2007). Foram utilizados métodos explícitos usados para identificar, selecionar e incluir estudos conforme critérios predefinidos, e na sequencia os resultados dos estudos foram integrados e comparados, conforme sugerido por LIBERATI et al. (2009) e MOHER et al. (2009). A revisão sistemática foi realizada em três estágios que compreendem: 1) Planejamento; 2) Condução; e, 3) Apresentação dos resultados.

\subsection{Planejamento}

Nesta etapa, é apresentada a justificativa da pesquisa, seguida do desenvolvimento de um protocolo de revisão. O tema sugerido para este estudo aborda os 'fatores de exposição e seus efeitos na SST de pilotos agrícolas'. Em uma pesquisa inicial em vários mecanismos de busca, não foram encontradas revisões 
sobre fatores de exposição. Isto justifica a necessidade para resumir toda a informação existente sobre este tema de forma completa e imparcial, como primeiro passo para outras atividades de investigação. Os dados resultantes da investigação sobre os fatores de exposição podem identificar eventuais lacunas de pesquisa, e sugerir áreas específicas para investigação adicional. Baseado nesta necessidade criou-se um protocolo de pesquisa, onde se procurou identificar: 'quais são os fatores de exposição suportados pelos pilotos agrícolas e quais são os efeitos causados por estes fatores na SST?' Esta questão de pesquisa limita o estudo a um cenário específico, sob a perspectiva de fatores humanos, na avaliação de fenômenos oriundos da interação do homem com seu trabalho. Através desta questão, procura-se responder sobre: 1) O problema, sendo os fatores de exposição; 2) Os efeitos, resultado da ação dos fatores de exposição e o comprometimento da SST para os pilotos agrícolas. Para isto, realizou-se uma pesquisa piloto em diversas bases de dados, através de diversos mecanismos de busca (ou sistemas informatizados de busca online), utilizando palavras-chave normalmente encontradas nos estudos sobre aviação agrícola e SST com base nos conhecimentos prévios dos autores. Com os resultados destas buscas, foram estabelecidos: 1) As bases de dados e os mecanismos de busca; 2) As palavras chaves; 3) Os algoritmos de busca; e, 4) Procedimentos de busca. Como mecanismos de busca, foram utilizados: 1) 'PubMed' (disponível em: http://www.ncbi.nlm.nih.gov/pubmed); 2) 'Science Direct' (disponível em: http://www.sciencedirect.com); e 3) 'ISI Web of Knowledge' (disponível em: http://apps.webofknowledge.com). Para resultar inicialmente em um maior número possível de artigos da área, foram utilizados como base para a seleção das palavras-chave: 1) O setor de aplicação, incluindo as palavras: 'agricultural' e 'aviation'; e, 2) A atividade realizada, definida pelo termo: 'aerial application'. Essas palavras-chave foram utilizadas conforme o seguinte padrão de algoritmo de busca avançada: (("aerial application”) OR (agricult* AND aviat*)). Este padrão de algoritmo foi modificado conforme manual de aplicação de cada um dos mecanismos de busca, e posteriormente aplicado nos campos que compreendem: 1) Título; 2) Palavras-chave; e, 3) Resumo, de forma retrospectiva a partir de 2011. Os 
resultados compreenderam todos os estudos descritivos, explicativos e exploratórios sobre qualquer assunto que compreende a aviação agrícola e a agricultura.

\subsection{Condução}

A pesquisa foi realizada conforme os procedimentos de busca definidos no planejamento da revisão, com as devidas alterações nos algoritmos de busca, de acordo com as limitações de cada mecanismo de busca, resultando inicialmente em 911 artigos. A partir destes resultados, foram aplicados os seguintes critérios de exclusão: 1) Quanto ao idioma de publicação, foram desconsiderados os artigos com texto completo indisponível em língua inglesa; 2) Quanto à tipologia, foram descartados materiais editoriais, livros, capítulos de livros e literatura cinzenta (refere-se ao que é produzido em todos os níveis do governo, institutos, academias, empresas e indústria, em formato impresso e eletrônico, mas que não é controlado por editores científicos ou comerciais), mantendo apenas estudos peer reviewed. Estes critérios foram aplicados através de filtros informatizados, quando disponíveis nos mecanismos de busca, e que resultaram na exclusão de 95 artigos. Em seguida, foi realizada uma avaliação de elegibilidade, de forma manual, padronizada, através da leitura dos campos que compreendem: 1) palavras-chave; 2) título; e, 3) resumo. Foram estabelecidos os seguintes critérios de inclusão: 1) Quanto à abordagem, foram considerados apenas os estudos apresentando resultados sobre a 'aviação agrícola', incluindo sinônimos (operações aeroagrícolas, aplicação aérea, pulverização aérea). Foram considerados nesta etapa, estudos sobre 'operações aéreas com carga externa' que podem estar associados aos objetivos desta pesquisa; 2) Quanto aos indivíduos estudados, foram compreendidos 'pilotos agrícolas', incluindo sinônimos (pilotos de carga externa, pilotos aplicadores) Foram considerados nesta etapa, estudos que apresentaram resultados sobre outros 'profissionais de apoio', por vezes referidos como 'equipe de terra', 'tripulação terrestre', e 'equipe de apoio'. A informação qualitativa sobre estes outros profissionais foi considerada de forma isolada; 3) Quanto ao objetivo, foram considerados estudos explicativos, descritivos ou exploratórios sobre 'fatores de exposição', que podem contribuir para agravar as condições de SST. Esta etapa 
resultou na exclusão de 775 artigos. Dos 41 artigos restantes, 19 duplicados foram excluídos, resultando em 28 artigos para solicitação de texto completo. Estes artigos serviram para uma segunda pesquisa bibliográfica, baseada nas referências citadas. Os artigos referenciados foram selecionados segundo os mesmos critérios de inclusão e exclusão utilizados anteriormente, porém limitados somente ao título do estudo. Foram incluídos 12 artigos durante a pesquisa bibliográfica, que também foram solicitados para leitura, e juntamente com os artigos oriundos da busca booleana foram analisados quanto ao conteúdo, onde foram extraídas as informações qualitativas. Essa etapa foi realizada de forma independente por dois pesquisadores e as divergências foram esclarecidas em consenso conforme apontado por LIBERATI et al. (2009). A sequência lógica dos procedimentos de busca, assim como os resultados que apresentam a seleção dos estudos estão representados na figura 1.

\subsection{Apresentação dos resultados}

A informação qualitativa dos estudos foi extraída de cada estudo incluindo: 1) O foco do estudo, sendo a um fator de exposição 'específico' ou ao 'ambiente de trabalho como um todo', incluindo os efeitos da exposição a vários fatores; 2) Indivíduos expostos (pilotos e equipe de apoio); 3) Principais resultados dos estudos (efeitos causados pelos fatores de exposição na SST); 4) Limitações apresentadas nos estudos considerados; e, 5) Lacunas de pesquisa identificadas nos estudos considerados. Ainda com relação ao foco do estudo, estes foram categorizados de acordo com o fator de exposição, baseado em um modelo adaptado de MORAES \& MONT'ALVÃO (2000), onde foram excluídos alguns fatores que não se aplicam na aviação. Os fatores de exposição incluídos são os seguintes: 1) Fatores organizacionais (objetivação, responsabilidades, autonomia e participação); 2) Fatores acidentários (condições de pista, condições de voo, manutenção de aeronave e dispositivos, deficiência de rotinas e equipamentos para controle de acidentes e incêndios, falta de dispositivos de proteção, que incluem Equipamentos de Proteção Individual - EPI e Equipamentos de Proteção Coletiva - EPC); 3) 
Figura 1 - Procedimentos e resultados do processo de busca e seleção de estudos

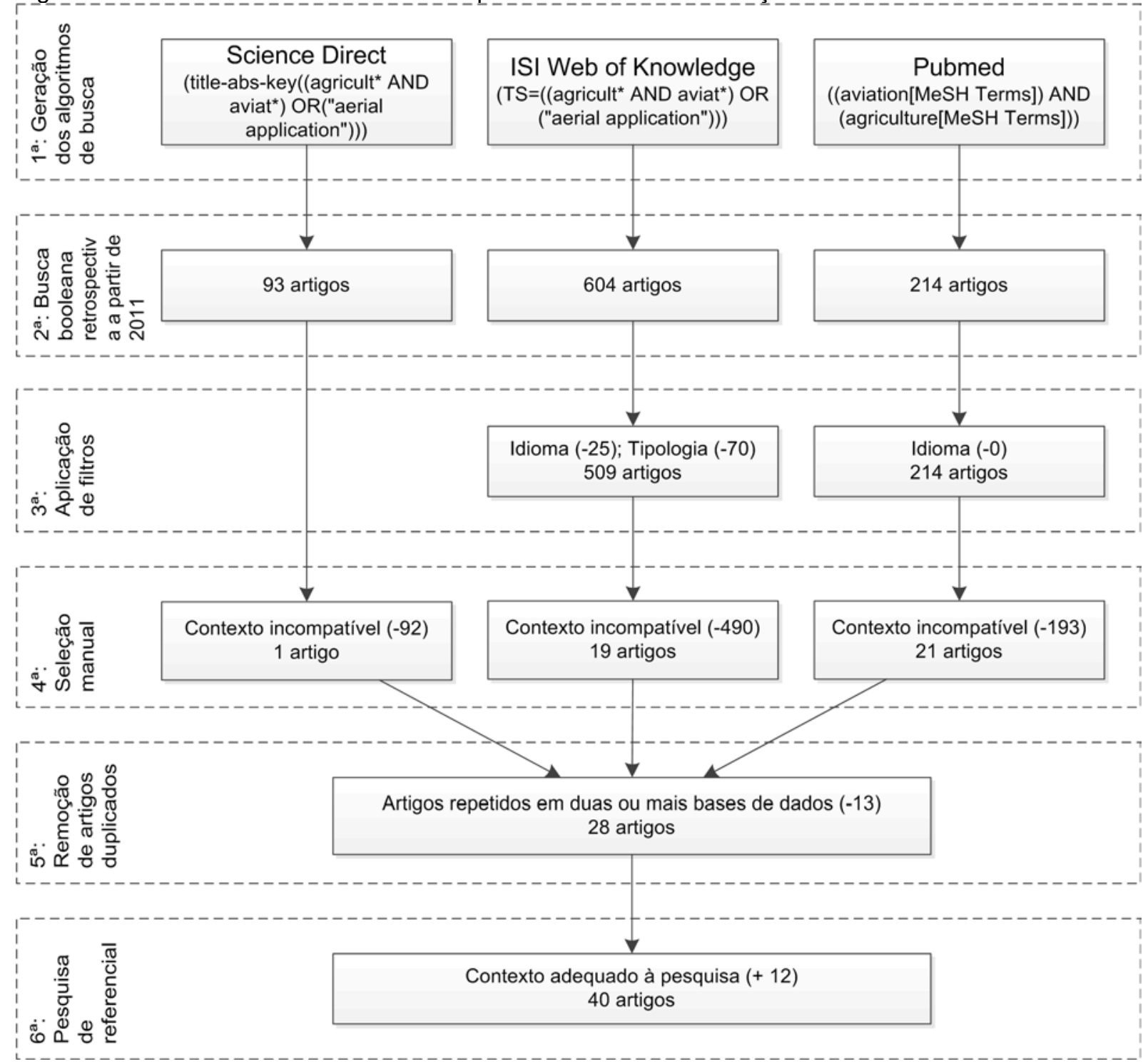

Fatores naturais (condições meteorológicas desfavoráveis para execução do trabalho); 4) Fatores físico-ambientais (ruído, vibração, iluminação, temperatura, forças gravitacionais); 5) Fatores químico-ambientais (partículas ou elementos tóxicos e aerodispersóides); 6) Fatores operacionais (ritmo de trabalho, repetitividade, monotonia, pressão por prazos de produção e controle); 7) Fatores cognitivo-informacionais (deficiência na detecção, discriminação ou identificação em instrumentos e mostradores, sinalização inadequada); 8) Fatores comunicacionais Revista Produção Online, Florianópolis, SC, v.15, n. 4, p. 1195-1223, out./dez. 2015. 
(Falta de dispositivos de comunicação à distância, ruídos na transmissão ou recepção de informações sonoras ou gestuais); 9) Fatores psicossociais (conflitos entre indivíduos ou grupos sociais, dificuldade de comunicação e interação interpessoal, falta de opção de descanso, alimentação, distração e laser no ambiente de trabalho); 10) Fatores acionais (Constrangimentos biomecânicos, e dimensionamento do posto de trabalho). Para a apresentação, foram reunidos alguns fatores de exposição que, de acordo com a informação recuperada, possuem efeitos semelhantes na SST dos pilotos agrícolas.

\section{RESULTADOS}

Através dos estudos selecionados pelos procedimentos de busca (Figura 1), foram identificados dois tipos de abordagens: 1) As que apresentaram de forma conjunta os fatores de exposição presentes no ambiente de trabalho; 2) As que apresentaram de forma 'isolada' os dados sobre um único fator de exposição.

A síntese dos dados de estudos que avaliaram o ambiente de trabalho (Quadro 1) apresenta as características e/ou os fatores de exposição suportados pelos pilotos agrícolas, que devem ser considerados em estudos sobre SST na aviação agrícola. Os fatores de exposição apresentados de forma conjunta permitem gerar soluções para o 'sistema de trabalho', onde a mitigação dos problemas de SST pode ser feita através da redução da fadiga física e mental, gerada pela soma dos perigos presentes no ambiente de trabalho.

A síntese dos dados de estudos focados em um único fator de exposição (Quadro 2), apresentam dados avaliados em profundidade que permitem compreender as relações entre a exposição e os efeitos na SST. Estes estudos são importantes porque evidenciam os perigos induzidos pelos fatores de exposição apresentados e sua potencialização em função da exposição a outros fatores considerados contribuintes. Além disto, os fatores de exposição estudados de forma isolada permitem gerar soluções pontuais para mitigação de problemas na SST dos pilotos agrícolas. 
Quadro 1 - Dados dos estudos dedicados à descrição do ambiente de trabalho como um todo, relacionando várias características e/ou fatores de exposição

\begin{tabular}{|c|c|c|}
\hline AUTOR & CONTEXTO & $\begin{array}{l}\text { FATORES } \\
\text { CONSIDERADOS }\end{array}$ \\
\hline (BARUCH, 1970) & $\begin{array}{l}\text { Investiga os fatores que são susceptíveis de } \\
\text { causar fadiga e reduzir alerta entre os pilotos e, } \\
\text { assim, contribuir para o aumento do número de } \\
\text { acidentes. }\end{array}$ & $\begin{array}{l}\text { QUA; OPR; NAT; } \\
\text { ACD; FIA. }\end{array}$ \\
\hline $\begin{array}{l}\text { (BILLINGS, } \\
1963)\end{array}$ & $\begin{array}{l}\text { Tentativa de evidenciar problemas clínicos de } \\
\text { saúde e ambientais verificados na aviação } \\
\text { agrícola. }\end{array}$ & $\begin{array}{l}\text { ACD; COM; FIA; INF; } \\
\text { NAT; OPR; ORG; } \\
\text { PSC; QUA. }\end{array}$ \\
\hline $\begin{array}{l}\text { (GORDON \& } \\
\text { HIRSCH, 1986) }\end{array}$ & $\begin{array}{l}\text { Mostra que a introdução de novos pesticidas } \\
\text { exige um monitoramento médico mais } \\
\text { desenvolvido e adequado. }\end{array}$ & 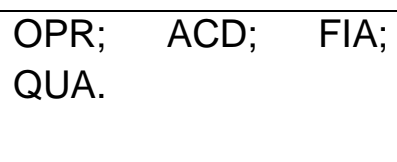 \\
\hline (HALL, 1991) & $\begin{array}{l}\text { Explora as atividades e atitudes que precedem } \\
\text { o acidente. }\end{array}$ & ACD; ORG; PSC \\
\hline $\begin{array}{l}\text { (NORTH \& } \\
\text { HIGHTOWER, } \\
\text { 1957) }\end{array}$ & $\begin{array}{l}\text { Apresentação do perfil dos pilotos envolvidos } \\
\text { em quatro acidentes com aeronaves agrícolas, } \\
\text { possíveis causas e soluções. }\end{array}$ & ACD; ORG. \\
\hline (PERRY, 1969) & $\begin{array}{l}\text { Descrição de um programa para formação e } \\
\text { supervisão, considerando os fatores } \\
\text { contribuintes na ocorrência de acidentes. }\end{array}$ & ACD; OPR; ORG. \\
\hline $\begin{array}{l}\text { (QUANTICK, } \\
\text { 1979) }\end{array}$ & $\begin{array}{l}\text { Apresenta melhorias aplicadas em uma } \\
\text { concepção de aeronave exclusiva para uso em } \\
\text { operações aeroagrícolas. }\end{array}$ & ACD; INF; ACN. \\
\hline $\begin{array}{l}\text { (REICH \& } \\
\text { BERNER, 1968) }\end{array}$ & $\begin{array}{l}\text { Descoberta de semelhanças, tendências e } \\
\text { padrões em acidentes na aviação agrícola. }\end{array}$ & ACD; ORG; QUA. \\
\hline $\begin{array}{l}\text { (RICHTER et al., } \\
\text { 1981) }\end{array}$ & $\begin{array}{l}\text { Apresenta um programa para a prevenção de } \\
\text { mortes e ferimentos por acidentes de } \\
\text { aeronaves, de acordo com uma matriz de } \\
\text { opções para intervenções derivadas da } \\
\text { epidemiologia de acidentes rodoviários. }\end{array}$ & $\begin{array}{l}\text { QUA; } \quad \text { FIA; } \\
\text { ACD. }\end{array}$ \\
\hline $\begin{array}{l}\text { (RYAN \& } \\
\text { DOUGHERTY, } \\
\text { 1969) }\end{array}$ & $\begin{array}{l}\text { Análise de } 383 \text { acidentes em aeronaves } \\
\text { envolvidas em operações de aplicação aérea, } \\
\text { com base em relatórios de acidentes. }\end{array}$ & $\begin{array}{l}\text { Tipo de aeronave; } \\
\text { Experiência do piloto. }\end{array}$ \\
\hline
\end{tabular}

Legenda: $\quad \mathrm{ACD}=\mathrm{Acidentário;} \quad \mathrm{COM}=$ Comunicacional; $\quad \mathrm{FIA}=\mathrm{F}$ ísico-ambiental; $\quad \mathrm{INF}=$ Informacional; $\mathrm{NAT}=$ Natural; OPR=Operacional; ORG=Organizacional; $\mathrm{PSC}=\mathrm{Osicossocial;} \mathrm{QUA}=\mathrm{Químico-ambiental}$; $\mathrm{ACN}=$ Acional.

Revista Produção Online, Florianópolis, SC, v.15, n. 4, p. 1195-1223, out./dez. 2015. 


\begin{tabular}{|c|c|c|}
\hline FATOR & CARACTERIZAÇÂO (AUTOR) & FATORES RELACIONADOS \\
\hline ACD & $\begin{array}{l}\text { EPC (BRUGGINK et al., 1964; HAVEN, DE, } \\
\text { 1953); EPI (BRUGGINK et al., 1964) }\end{array}$ & Projeto da aeronave \\
\hline FIA & $\begin{array}{l}\text { Condições térmicas (GRIBETZ et al., 1980); } \\
\text { Ruído (TOBIAS, 1968) }\end{array}$ & $\begin{array}{l}\text { Falta de dispositivos de } \\
\text { proteção; uso incorreto de EPI; } \\
\text { e, gestão de ingestão de } \\
\text { líquidos. }\end{array}$ \\
\hline QUA & $\begin{array}{l}\text { Exposição aos químicos aplicados (ATALLAH et } \\
\text { al., 1982; CABLE \& DOHERTY, 1999; } \\
\text { CHATURVEDI, 2011; CHESTER \& WARD, } \\
\text { 1984; CHESTER et al., 1987; COHEN et al., } \\
\text { 1979; DAUGHERTY et al., 1962; DRISKELL et } \\
\text { al., 1991; GERRY et al., 2005; GORDON \& } \\
\text { RICHTER, 1991; GUPTA et al., 1980; KNARR } \\
\text { et al., 1985; KNOPP \& GLASS, 1991; MINASI et } \\
\text { al., 2011; QUANTICK \& PERRY, 1981; RICE et } \\
\text { al., 2005; RICHTER et al., 1980; ROAN et al., } \\
\text { 1984; SMITH, 1963; ULLMANN et al., 1979; } \\
\text { WOOD et al., 1971; YOSHIDA et al., 1990); } \\
\text { Exposição aos gases de combustão da } \\
\text { aeronave (CHATURVEDI, 2011). }\end{array}$ & $\begin{array}{l}\text { Uso incorreto de EPI; falta de } \\
\text { EPC; obstáculos; } \\
\text { procedimentos realizados de } \\
\text { forma incorreta; qualificação } \\
\text { profissional; } \\
\text { meteorológicas desfavoráveis; } \\
\text { forças gravitacionais; condições } \\
\text { térmicas desfavoráveis; ritmo } \\
\text { de trabalho intenso; atitude e } \\
\text { experiência dos profissionais; e, } \\
\text { exposição direta devido aos } \\
\text { acidentes. }\end{array}$ \\
\hline ORG & $\begin{array}{l}\text { Gestão da equipe (VOOGT, DE, } \\
\text { UITDEWILLIGEN e EREMENKO, 2009); } \\
\text { Gestão da saúde (MOHLER, 1980). }\end{array}$ & $\begin{array}{l}\text { Apresentação das informações; } \\
\text { comunicação entre pilotos e } \\
\text { equipe de solo; e, } \\
\text { responsabilidades centralizadas } \\
\text { no piloto. }\end{array}$ \\
\hline
\end{tabular}

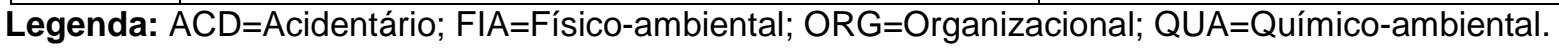

\subsection{Fatores organizacionais, operacionais e psicossociais}

Para os fatores psicossociais, organizacionais, operacionais, vários dos efeitos são semelhantes e muitas vezes contribuintes para ocorrência de acidentes. Pressões por prazos (HALL, 1991), atitudes e responsabilidades (HALL, 1991), ritmo de trabalho (CANTOR \& SILBERMAN, 1999; GORDON \& HIRSCH, 1986; HALL, 1991), condições de trabalho (YOSHIDA et al., 1990), distanciamento da família (HALL, 1991) entre outros, têm efeitos psicológicos que podem causar perda de desempenho (HALL, 1991) e aumento da fadiga (GORDON \& HIRSCH, 1986). Estes 
efeitos podem comprometer as tomadas de decisão, importantes para a segurança das operações.

Fatores organizacionais foram apresentados como parte dos problemas presentes no ambiente de trabalho dos pilotos agrícolas (BILLINGS, 1963; HALL, 1991; REICH \& BERNER, 1968; VOOGT et al., 2009), ou como fatores relacionados com a exposição aos fatores físico-ambientais (GRIBETZ et al., 1980) e químicoambientais (CABLE \& DOHERTY, 1999; CHESTER et al., 1987; QUANTICK \& PERRY, 1981), devido aos procedimentos realizados de forma incorreta, que podem ocasionar exposição adicional. Os fatores organizacionais são apresentados principalmente no que diz respeito às responsabilidades impostas aos pilotos, podendo ser durante a execução da aplicação propriamente dita ou atividades relacionadas. Durante a aplicação, o piloto deve realizar a condução da aeronave que inicialmente está carregada, decidir sobre a abordagem de cada campo, tendo em conta os ventos predominantes e a localização das culturas adjacentes, que podem ser prejudicadas por suas substâncias químicas (BILLINGS, 1963). Esta abordagem é feita através de aplicação de comandos realizados com ambas as mãos e pés, e com controle de trajetória realizado através de mostradores de velocidade do ar, altitude e direção. No final de cada aplicação ele deve pousar a aeronave nas proximidades do campo, geralmente em pistas improvisadas, já estando sem carga.

Em alguns estudos, são apresentadas as equipes de terra para auxiliar o piloto agrícola em etapas de reabastecimento e carregamento da aeronave. Contudo, em alguns estudos, os procedimentos são realizados pelos próprios pilotos, como no caso de pilotos que atuam na aplicação de produtos para controle de vetores (ser vivo invertebrado capaz de transmitir de forma ativa ou passiva um agente infectante), mesmo necessitando uma qualificação profissional diferente. Em teoria, quando existe a tripulação terrestre, o piloto não entra em contato direto com produtos e equipamentos, aguardando dentro da cabine da aeronave. Em alguns estudos, parte da responsabilidade pelo posicionamento é da equipe de solo, feita por um profissional também chamado de 'bandeirinha', que foi substituído pelos Sistemas de Posicionamento Global Diferencial (DGPS - Differential Global Positioning System). Apesar do apoio da equipe de terra, cabe ao piloto gerenciar a 
operação durante todo o trabalho, incluindo a responsabilidade sobre estes profissionais de apoio (BILLINGS, 1963).

Quanto às outras atividades, indiretamente ligadas à aplicação, podemos citar a atuação em processos decisórios sobre a aplicação, geralmente feita com a presença de um engenheiro agrônomo e o proprietário da propriedade rural. $\mathrm{O}$ trabalho de piloto agrícola requer alta qualificação (VOOGT et al., 2009), vistas todas as responsabilidades citadas, que são ainda acompanhadas da atuação como vendedor de seus serviços e gestão da empresa quando estes profissionais trabalham de forma autônoma, ou gerenciando pequenas empresas de aplicação aérea (BILLINGS, 1963).

Fatores operacionais são apresentados no contexto da maioria dos estudos sobre fatores de exposição na aviação agrícola. A pressão por prazos é considerada como problema relacionado à SST de pilotos agrícolas desde os anos 60's (BILLINGS, 1963), na minimização dos tempos improdutivos, como carregamento da aeronave e a execução de manobras. O ritmo de trabalho é presente principalmente devido à sazonalidade das operações, e o piloto agrícola pode inclusive trabalhar nestes períodos acima do limite recomendado (BILLINGS, 1963). A sazonalidade pode inclusive fazer com que o piloto agrícola trabalhe o suficiente durante os meses de maior demanda para se sustentar durante todo o resto do ano (BILLINGS, 1963). A pressão por prazos, o ritmo de trabalho e a concorrência entre as empresas de aplicação aérea criam uma resistência às alterações do turno de trabalho, independentemente de qualquer regulamentação que possa ser imposta (BILLINGS, 1963). Como efeitos destes fatores operacionais, está o aumento do tempo em que o trabalhador está exposto a outros fatores como os químico-ambientais (RICHTER et al., 1980).

Fatores psicossociais foram apresentados como parte dos problemas presentes no ambiente de trabalho dos pilotos agrícolas apenas em dois dos estudos incluídos nesta revisão (BILLINGS, 1963; HALL, 1991). Estes fatores não aparecem como contribuintes em nenhum estudo dedicado a outros fatores, nem no contexto dos mesmos. Como maior preocupação, pode-se citar o distanciamento da família, que tem efeitos psicológicos que podem ocasionar a perda de desempenho (HALL, 1991), e assim comprometer a segurança das operações. 


\subsection{Fatores naturais e acidentários}

Os estudos que abordam os fatores naturais e os fatores acidentários incluídos nesta revisão, em sua maioria, apresentam estes diretamente relacionados à organização do trabalho, principalmente no que diz respeito às relações entre as condições de trabalho e os procedimentos realizados pelo piloto agrícola. Estes fatores foram considerados conjuntamente porque se confundem na bibliografia, que os trata de forma complementar, geralmente como perigos associados aos acidentes de trabalho e não à saúde ocupacional.

Fatores naturais foram apresentados como parte dos problemas presentes no ambiente de trabalho dos pilotos agrícolas (BARUCH, 1970; BILLINGS, 1963). Situações de perigo incluem o voo em condições de vento desfavoráveis, que compromete a segurança do piloto nas manobras, e no desvio da atenção do piloto para o controle da deriva do produto para áreas inapropriadas (BILLINGS, 1963). Estas condições podem se tornar um perigo, principalmente quando potencializadas por outros fatores como o ruído ambiental (TOBIAS, 1968), que podem prejudicar a atenção do piloto, e a fadiga e a pressão por prazos, que podem alterar a resposta de um piloto para negação de trabalho (HALL, 1991) em condições meteorológicas desfavoráveis (BILLINGS, 1963).

Fatores acidentários foram apresentados desde os estudos dos anos 60's, até os mais recentes (BARUCH, 1970; BILLINGS, 1963; GORDON \& HIRSCH, 1986; HALL, 1991; REICH \& BERNER, 1968; RICHTER et al., 1980; RYAN \& DOUGHERTY, 1969; VOOGT et al., 2009). Situações de perigo criadas pela exposição aos fatores acidentários incluem a falta de EPC, o uso incorreto de EPI (ou até mesmo a falta destes), péssimas condições de pista e obstáculos presentes no trajeto da aeronave. Com relação aos EPC, pode-se dizer que uma grande evolução iniciou a partir dos anos 60's. Neste período, as aeronaves militares adaptadas para uso agrícola começaram a ser substituídas por outras especialmente desenvolvidas para uso nestas operações (BRUGGINK et al., 1964). Estas aeronaves, já contemplavam em seus projetos o tipo de atividade executada e os perigos envolvidos. A falta do uso, ou uso inadequado de EPI e EPC pode contribuir para a exposição aos produtos químicos aplicados (MINASI et al., 2011; 
RICHTER et al., 1980) e ao ruído ambiental (TOBIAS, 1968). A ineficiência dos EPI e EPC pode estar associada também a durabilidade e falta de inspeção (BILLINGS, 1963; TOBIAS, 1968). Os EPIs insatisfatórios são apontados como uma área fecunda para investigação e atuação (BILLINGS, 1963), contudo, deve haver muito cuidado no estudo sobre EPI, visto que o uso de certos equipamentos pode melhorar as condições relacionadas a um determinado fator de exposição e ao mesmo tempo prejudicar a exposição a outro. Como exemplo disto, o uso de macacão que pode dificultar a exposição aos fatores químico-ambientais (ATALLAH et al., 1982) porque impede que o contato com os agentes químicos, ao mesmo tempo que a falta de inspeção e limpeza deste EPI pode facilitar esta contaminação (YOSHIDA et al., 1990). Além disto, o uso do macacão pode aumentar a temperatura corporal (FÆREVIK \& REINERTSEN, 2010), se não usados em conjunto com sistemas de ventilação como o ar-condicionado. Outros fatores acidentários como: baixa altitude do voo com o perigo sempre presente de obstáculos inesperados (BARUCH, 1970; BILLINGS, 1963; CANTOR \& SILBERMAN, 1999; HALL, 1991), com decolagem e pouso em pistas geralmente improvisadas (HALL, 1991), aparecem durante toda a história da aviação agrícola, mostrando que as mudanças são insuficientes para controlar os acidentes de trabalho.

\subsection{Fatores físico-ambientais e químico-ambientais}

Fatores físico-ambientais foram apresentados como parte dos problemas presentes no ambiente de trabalho dos pilotos agrícolas (BARUCH, 1970; BILLINGS, 1963; GORDON \& HIRSCH, 1986; RICHTER et al., 1981). Entre os principais fatores, estão o ruído, condições térmicas, choques, vibrações e forças gravitacionais. Este conjunto de fatores pode aumentar significativamente a fadiga do piloto (BILLINGS, 1963), levando-o a condições de desconforto e redução do desempenho que podem em curto prazo comprometer a segurança e, em longo prazo ter efeitos sobre a saúde do piloto. O ruído em altas intensidades e durante grande período de tempo pode causar Perda Auditiva Induzida pelo Ruído (PAIR), que por sua vez pode comprometer a atenção do piloto, a recepção de estímulos 
auditivos e sinais de alerta (TOBIAS, 1968). A temperatura da cabine pode comprometer as condições fisiológicas, causando perda de massa corporal, desidratação, alteração da pressão sanguínea, alteração da temperatura central (GRIBETZ et al., 1980), e em casos mais graves a exposição ao calor pode causar perda de vigilância, que podem aumentar o risco de erro do piloto (F/EREVIK \& REINERTSEN, 2010). As vibrações, assim como choques e forças gravitacionais, são citadas em alguns estudos da aviação agrícola (GRIBETZ et al., 1980; RICHTER et al., 1980), enquanto seu conhecimento de forma mais aprofundada não foi visto em nenhum estudo apresentado nesta revisão.

Fatores químico-ambientais também foram apresentados como parte dos problemas presentes no ambiente de trabalho dos pilotos agrícolas (BARUCH, 1970; BILLINGS, 1963; GORDON \& HIRSCH, 1986; REICH \& BERNER, 1968; RICHTER et al., 1981). Além destes, vários autores realizaram estudos para compreender as relações entre estes fatores e a segurança (CABLE \& DOHERTY, 1999; CHESTER et al., 1987; DAUGHERTY et al., 1962; DRISKELL et al., 1991; GERRY et al., 2005; GORDON \& RICHTER, 1991; KNOPP \& GLASS, 1991; MINASI et al., 2011; QUANTICK \& PERRY, 1981; RICHTER et al., 1980; ROAN et al., 1984). Existe a evidência que a exposição do piloto para produtos químicos tóxicos pode desempenhar um papel contributivo ou causal em acidentes na aviação agrícola (REICH \& BERNER, 1968). Esta exposição pode causar a contaminação do piloto, que pode ocorrer por via cutânea (ATALLAH et al., 1982; CHESTER et al., 1987; RICHTER et al., 1980; YOSHIDA et al., 1990), respiratória (ATALLAH et al., 1982) e ingestão (DRISKELL et al., 1991). A exposição por via respiratória pode ser aumentada devido a condições de organização do trabalho, devido às manobras de ida e volta, efetuadas em algumas situações para redução de tempo improdutivo (BILLINGS, 1963). Assim na volta, o piloto fica exposto a partículas presentes no ar que não tiveram tempo suficiente para se depositar no alvo. A exposição pode também ser aumentada por fatores acidentários como a falta de uso de EPI (GERRY et al., 2005; MINASI et al., 2011; RICHTER et al., 1980) e falta de EPC (GORDON \& RICHTER, 1991; RICHTER et al., 1980). A exposição por via cutânea pode ocorrer quando existem falhas no isolamento da cabine com a parte exterior da aeronave, permitindo a entrada de partículas e o contato com a pele do piloto agrícola 
(RICHTER et al., 1980). Esta condição também é prejudicada na falta de uso de vestimentas adequadas (YOSHIDA et al., 1990) e em condições térmicas desfavoráveis (FÆREVIK \& REINERTSEN, 2010), quando o suor aumenta a absorção da pele. A contaminação por ingestão ocorre em situações de acidente, quando a colisão com obstáculos, normalmente a baixas velocidades, não permite o alijamento do produto químico (QUANTICK \& PERRY, 1981), e este entra em contato direto com o piloto agrícola (CABLE \& DOHERTY, 1999; DRISKELL et al., 1991; QUANTICK \& PERRY, 1981; TESTUD \& BOUGON, 2009). Estes compostos, absorvidos sob qualquer via, prejudicam o desempenho neurológico de alto nível de precisão exigido para voar (DELLINGER \& TAYLOR, 1985; QUANTICK \& PERRY, 1981; REICH \& BERNER, 1968; RICHTER et al., 1981), prejudicando assim a segurança das operações. Tecnologias mais recentes como os produtos químicos de ultra baixo volume (ULV - Ultra Low Volume) parecem resolver grande parte dos problemas de contaminação (GERRY et al., 2005; YOSHIDA et al., 1990). Isto porque os compostos ULV não exigem diluição no campo, o que significa menor contato com o produto químico. Além disto, como se trata de um produto extremamente concentrado, exige equipamentos especiais com maior controle de processo.

\subsection{Fatores informacionais e comunicacionais}

Fatores informacionais e comunicacionais foram apresentados apenas como parte dos problemas presentes no ambiente de trabalho dos pilotos agrícolas em dois dos estudos incluídos nesta revisão (BILLINGS, 1963; VOOGT et al., 2009). Na década de 60, um argumento de pilotos agrícolas experientes era de que não existiam instrumentos capazes de ajudar o piloto agrícola, caso ele não esteja intimamente familiarizado com todos os aspectos e limitações de desempenho do seu avião (BILLINGS, 1963). Como por exemplo, os instrumentos existentes informativos de velocidade do ar são relativamente insatisfatórios, visto que o piloto raramente os observa durante as manobras. Isto, devido às exigências de atenção que imperam com relação às manobras voltadas para o campo, em função da 
continuação do seu plano de voo. Estudos atuais não abordam estes problemas, sendo difícil constatar alguma evolução presente em estudos científicos.

Também na década de 60, acrescentava-se uma série de equipamentos de comunicação, acompanhados de diversos recursos que estavam disponíveis no mercado poderiam ser listados, sendo necessário somente a adaptação e utilização nas aeronaves agrícolas (BILLINGS, 1963). Nos tempos atuais, onde estas condições parecem ter sido parcialmente solucionadas, e um estudo mais recente analisa a comunicação entre piloto e equipe adicional de voo ou de terra, que pode ajudar o piloto a manter a vigia visual (VOOGT et al., 2009). Entre as principais conclusões sobre a comunicação entre piloto e equipe de apoio, tem-se que o número de acidentes poderia ser ainda mais reduzido com uma gestão de combustível (ou controle de consumo e abastecimento) adequada em que o piloto e equipe de terra poderiam contribuir um com o outro (VOOGT et al. 2009). Além disto, melhores equipamentos pessoais, incorporando equipamentos de comunicação à distância, são apresentados como possíveis soluções, que tornariam a tarefa do piloto mais fácil e, assim, ajudariam a aliviar a fadiga (BILLINGS, 1963).

\section{DISCUSSÃO}

Nesta discussão, os autores procuraram comparar os dados dos estudos incluídos nesta revisão, além de colocar suas opiniões baseadas no conhecimento do tema e do setor estudado. O objetivo disto é preencher certas lacunas temporais e permitir uma compreensão mais clara sobre a evolução das tecnologias presentes no ambiente de trabalho dos pilotos agrícolas e a possível melhoria das condições de trabalho.

\subsection{Evolução temporal dos estudos sobre os fatores humanos e a segurança e saúde ocupacional dos pilotos agrícolas}

Devido à inclusão de diversos estudos, muitos destes antigos, torna-se necessária uma avaliação mais detalhada sobre quais destes fatores ainda contribuem de forma importante para o comprometimento da SST dos pilotos agrícolas. Isto porque várias tecnologias surgiram durante este período (ex. Revista Produção Online, Florianópolis, SC, v.15, n. 4, p. 1195-1223, out./dez. 2015. 
Melhorias estruturais de aeronaves (BRUGGINK et al. 1964; HAVEN, 1953), ULV (GERRY et al., 2005; YOSHIDA et al., 1990), etc.) e muitas situações críticas citadas em alguns estudos podem não refletir mais as condições atuais, como exemplo, a elevada ocorrência de traumas graves (REICH \& BERNER, 1968; RYAN \& DOUGHERTY, 1969) e a perda de desempenho ocasionada pela exposição química (BILLINGS, 1963), para citar alguns.

A aviação agrícola foi definida em estudo da década de 60 como a forma mais perigosa de voo, mesmo considerando os avanços tecnológicos (BRUGGINK, et al., 1964), devido aos riscos presentes na realização do trabalho (BILLINGS, 1963). Neste período, iniciou-se a preocupação com os possíveis problemas relacionados à segurança e saúde ocupacional, inicialmente com a mudança nas características estruturais dos aviões militares antigos adaptados, para projetos mais específicos para o trabalho agrícola (BRUGGINK et al., 1964; HAVEN, 1953). Isto também ficou evidenciado por estudos que tentaram levantar as características (RYAN \& DOUGHERTY, 1969) e fatores de exposição (BILLINGS, 1963; REICH \& BERNER, 1968) presentes no ambiente de trabalho. A partir destes dados, surgiram outros estudos dedicados a fatores de exposição específicos, como químicoambientais (DAUGHERTY et al., 1962) e acidentários (BRUGGINK et al., 1964). No entanto, fatores operacionais (SMITH et al., 1968), organizacionais e comunicacionais (REICH \& BERNER, 1968) foram somente abordados como possíveis fatores contribuintes para ocorrência de acidentes de trabalho.

A partir da década de 70, percebeu-se uma preocupação maior com outros fatores de exposição, abordando os efeitos da carga de trabalho, e condições ambientais na tentativa de explicar acidentes que até então pareciam inexplicáveis (BARUCH, 1970). Surgiram alguns estudos sobre fatores físico-ambientais, pouco abordados até então, principalmente quanto às condições térmicas (BARUCH, 1970; GRIBETZ et al., 1980). Além disto, no contexto dos estudos são apresentados como fatores contribuintes para perda de desempenho dos pilotos as forças gravitacionais, vibrações e ruído (GRIBETZ et al., 1980). Outros estudos ainda consideraram em seu contexto outros fatores como os operacionais e organizacionais (BARUCH, 1970), compreendendo principalmente a carga de trabalho e a falta repouso. 
Embora a preocupação com vários fatores de exposição, e da relação entre eles com problemas de SST, a partir da década de 80, os estudos se concentraram quase que essencialmente nos fatores químico-ambientais. Estes estudos foram justificados pela vasta utilização de produtos tóxicos (ATALLAH et al., 1982; QUANTICK \& PERRY, 1981; RICHTER et al., 1980), que evoluíram de forma rápida na década de 80 para suprir as necessidades da agricultura em aplicações terrestres e aéreas (GORDON \& HIRSCH, 1986).

Nos estudos mais recentes, a partir da década de 90, passou-se a ter uma preocupação com as atividades e atitudes que precedem o acidente (HALL, 1991), tentando compreender as reais causas dos acidentes. Além disto, outros fatores até então considerados apenas como contribuintes, como os informacionais e comunicacionais, foram abordados como causa raiz nestes acidentes (VOOGT et al., 2009). Neste contexto, a maioria dos estudos apresentou resultados focados apenas em relação aos fatores químico-ambientais (CABLE \& DOHERTY, 1999; DRISKELL et al., 1991; GORDON \& RICHTER, 1991; KNOPP \& GLASS, 1991; MINASI et al., 2011; YOSHIDA et al., 1990).

A falta de publicações sobre o ambiente de trabalho e a SST pôde ser observada desde a década de 60 , em que a literatura médica sobre aviação não refletia a importância deste segmento da indústria da aviação civil (BILLINGS, 1963). Embora muitas tecnologias tenham surgido até os dias atuais, a aviação agrícola continua sendo a forma mais perigosa de voo, e a literatura acadêmica ainda não reflete a importância da SST destes pilotos, sendo a atividade da aviação com menos estudos publicados sobre este tema (VOOGT et al., 2009).

\subsection{Relação entre fatores de exposição, segurança e saúde ocupacional}

No que concerne aos fatores de exposição apontados pelos estudos, é possível depreender que apenas alguns dos fatores comunicacionais, informacionais e psicossociais podem comprometer a segurança do trabalho isoladamente, sem interferências nas condições de saúde e desempenho. Isto porque esses fatores têm como principal a falta de atenção (ou desvio de atenção), que pode causar acidentes de trabalho, seja pela dificuldade na visualização de informação (REICH \& BERNER, 1968), nas distrações criadas através de sistemas de comunicação, e problemas de Revista Produção Online, Florianópolis, SC, v.15, n. 4, p. 1195-1223, out./dez. 2015. 
ordem pessoal (HALL, 1991), como problemas psicossociais (remuneração, afastamento da família, etc.) que podem resultar em falhas na identificação de perigos. Além destes, os fatores acidentários também podem ser causa direta de acidentes, e estão entre os mais notados, desde os primeiros estudos do setor, incluindo: obstáculos (REICH \& BERNER, 1968; RYAN \& DOUGHERTY, 1969; VOOGT et al., 2009), condições de pista (VOOGT et al., 2009) e manutenção (BRUGGINK et al., 1964).

Com relação à saúde ocupacional, praticamente todos os fatores tratados nos estudos incluídos nesta revisão têm colaboração na evolução de problemas de saúde, que podem também comprometer a segurança do trabalho quando em exposição prolongada. Contudo, deve-se ressaltar que os fatores mais prejudiciais apontados nos estudos desta revisão são os físico-ambientais e químico-ambientais.

Para os fatores químico-ambientais, as consequências mais graves estão ligadas à contaminação, que por sua vez, pode causar: fadiga (CANTOR \& SILBERMAN, 1999; GORDON \& HIRSCH, 1986; MOHLER, 1980; RICHTER et al., 1981; SMITH et al., 1968); problemas respiratórios (JONES et al., 2003); problemas cardiovasculares e termorreguladores (GRIBETZ et al., 1980); e, déficits neurológicos e psicomotores (CABLE \& DOHERTY, 1999; CANTOR \& SILBERMAN, 1999; DRISKELL et al., 1991; GORDON \& HIRSCH, 1986; TESTUD \& BOUGON, 2009). Estes, inicialmente ocasionam perda de desempenho que se torna perigoso, considerado o alto nível de precisão exigido no trabalho de piloto agrícola (DELLINGER \& TAYLOR, 1985; QUANTICK \& PERRY, 1981; REICH \& BERNER, 1968; RICHTER et al., 1981) e, desta forma, podendo contribuir para ocorrência de acidentes (CABLE \& DOHERTY, 1999; DRISKELL et al., 1991; TESTUD \& BOUGON, 2009). Para os fatores físico-ambientais, as consequências mais graves descritas pelos estudos estão ligadas à exposição a altas temperaturas que, além da fadiga, podem potencializar a exposição aos agentes toxicológicos. Além destes, a exposição a ruídos, vibrações, forças gravitacionais entre outros, também contribuem para o aumento da fadiga. Embora estes fatores não estejam representados nos resultados dos estudos incluídos nesta revisão, em alguns estudos da aviação militar, como em pilotos de helicópteros (BALASUBRAMANIAN et al., 2011; KÅSIN et al., 2011) e aviões de caça (AYDOG et al., 2004; SMITH, 
2004), indicam que seus efeitos podem ser danosos para saúde do piloto, comprometendo o sistema osteomuscular (dores e lesões na coluna vertebral) (AYDOG et al., 2004; BALASUBRAMANIAN et al., 2011; KÅSIN et al., 2011) e a recepção de informação (ressonância do globo ocular) (SMITH, 2004), podendo também afetar a segurança do trabalho (Perda de controle). Algumas atividades de trabalho na aviação agrícola sugerem que os pilotos possam estar expostos a intensidades semelhantes às apontadas nestes estudos militares, como por exemplo: o pouso e decolagem em pistas improvisadas, onde a presença de pequenos obstáculos como buracos e desníveis na pista podem gerar choques e vibrações.

É importante ainda considerar nos estudos sobre fatores físico-ambientais e químico-ambientais, que estes são potencializados por outros fatores incluindo: fatores acidentários (uso incorreto ou falta de EPI (BRUGGINK, BARNES e GREGG, 1964) e EPC (BRUGGINK et al., 1964; HAVEN, 1953)), que podem aumentar a exposição; fatores operacionais (ritmo de trabalho intenso (ATALLAH et al., 1982; COHEN et al., 1979; RICHTER et al., 1980; WOOD et al., 1971)), que aumentam o tempo de exposição, bem como os fatores organizacionais e/ou instrucionais (experiência e atitude (ATALLAH et al., 1982; RYAN \& DOUGHERTY, 1969; WOOD et al., 1971)), que estão relacionados a procedimentos incorretos.

Além destes fatores, podem-se citar os fatores comunicacionais (comunicação entre membros da equipe (VOOGT et al., 2009)), informacionais (qualidade e visualização da informação (VOOGT et al., 2009)) e acidentários (obstáculos e condições de pista (RICE et al., 2005; RICHTER et al., 1980)), que conjuntamente podem colaborar para perda de desempenho e contribuir para ocorrência de acidentes.

\subsection{Melhorias das condições de trabalho, soluções e respectivas restrições}

Recomendações para a melhoria das condições de trabalho para os pilotos agrícolas apresentadas nos estudos apresentados nesta revisão incluem: cabines isoladas, com ar condicionado e filtros; fornecimento de líquidos em abundância (GRIBETZ et al., 1980; RICHTER et al., 1980); gestão do tempo máximo diário de 
acordo com as condições de trabalho (RICHTER et al., 1980); remoção de obstáculos, sendo que quando não houver a possibilidade de retiradas destes (como no caso das linhas de energia elétrica), realizar uma sinalização clara (RICHTER et al., 1980); atribuir à equipe de apoio certas responsabilidades quanto à gestão de obstáculos, carga e combustível (VOOGT et al., 2009); realização de exames periódicos pelos pilotos agrícolas (GORDON \& HIRSCH, 1986) para prevenir de forma precoce a fadiga; e, instrução adequada (formação profissional) (BILLINGS, 1963).

O isolamento da cabine, assim como o uso de ar condicionado pode impedir a entrada de produtos químicos em geral (RICHTER et al., 1980; SAWINSKY \& PASZTOR, 1977), controlar a temperatura (GRIBETZ et al., 1980) e reduzir o ruído ambiental na cabine. Se este tipo de intervenção pode criar situações ideais (exposição abaixo dos limites estabelecidos por norma), estes fatores deixam de prejudicar a saúde do piloto agrícola, e de contribuir para o aumento da fadiga. Além disto, particularmente quanto à redução da exposição ao ruído, pode possibilitar o uso de informações sonoras independentes da atenção visual do piloto agrícola (BILLINGS, 1963).

A atribuição de responsabilidades para a equipe de apoio deve ser acompanhada de instrução adequada, assim como exigido para os pilotos agrícolas. As ações para gestão de horas de voo diárias requem maior conhecimento científico para poder comparar situações diferentes e quantificar quanto aos perigos existentes em cada situação.

Apesar das várias recomendações, é necessário ainda considerar que a exposição suportada pelos pilotos agrícolas é uma soma de todos os fatores presentes no ambiente de trabalho (PARSONS, 2000). Assim deve-se entender as relações entre os diferentes fatores de exposição.

\section{CONSIDERAÇÕES}

Neste estudo, foi apresentada uma revisão sistemática dos estudos que abordaram os fatores de exposição presentes no ambiente de trabalho dos pilotos agrícolas, e os efeitos destes na SST. Percebeu-se a existência de poucos estudos, 
apesar da importância deste tema para a agricultura, para aviação agrícola e para os profissionais envolvidos.

A abrangência de estudos mais antigos demonstrou as evoluções desenvolvidas visando suprir as necessidades do setor. Mesmo assim, a literatura atual não é suficiente para caracterizar e avaliar as condições de trabalho destes profissionais, visto que na maioria dos estudos, as informações são tratadas de forma superficial. Isto se reflete nas inúmeras lacunas de pesquisa, principalmente no que diz respeito a evolução das tecnologias, aeronaves e procedimentos operacionais.

A seguir são listadas algumas lacunas (informações não consolidadas) observadas neste estudo que servem inclusive como proposta para estudos futuros: 1) Quais as intensidades de exposição aos fatores físico-ambientais que os pilotos agrícolas estão expostos, e qual a eficácia dos EPIs e EPC utilizados para atenuar esta exposição? 2) Quais os constrangimentos posturais, acionais e comunicacionais criados no uso dos equipamentos presentes na cabine das aeronaves agrícolas? 3) Que intervenções podem minimizar os efeitos do afastamento da família na SST? 4) Que intervenções podem ser feitas para atenuar os efeitos dos fatores psicossociais (afastamento da família e relacionamento entre indivíduos de diferentes funções dentro da organização do trabalho)? 5) Qual a contribuição de cada fator de exposição para o aumento da fadiga do piloto agrícola? 6) Como realizar uma gestão de tempo de trabalho diário para diferentes ambientes de trabalho (ou diferentes fatores de exposição)?

\section{REFERÊNCIAS}

ATALLAH, Y. H.; CAHILL, W. P. \& WHITACRE, D. M. Exposure of pesticide applicators and support personnel to O-ethyl O-(4-nitrophenyl) phenylphosphonothioate (EPN). Archives of Environmental Contamination and Toxicology, v. 11, n. 2, p. 219-225, 1982. http://dx.doi.org/10.1007/BF01054899

ATSB. Aviation occurrence statistics: 2001 to 2010. Statistics. 2011.

AYDOG, S. T. et al. Cervical and lumbar spinal changes diagnosed in four-view radiographs of 732 military pilots. Aviation Space and Environmental Medicine, v. 75, n. 2, p. 154-157, 2004. 
BALASUBRAMANIAN, V.; DUTT, A. \& RAI, S. Analysis of muscle fatigue in helicopter pilots. Applied ergonomics, v. 42, n. 6, p. 913-8, 2011.

http://dx.doi.org/10.1016/j.apergo.2011.02.008

BARUCH, D. Some Medical Aspects in Agricultural Flights Relating to Fatigue Among Agricultural Pilots. Aerospace medicine, v. 41, n. 4, p. 447-450, 1970.

BILLINGS, C. E. Medical and environmental problems in agricultural aviation. Aerospace Medicine, v. 34, n. 5, p. 406-408, 1963.

BRUGGINK, G. M.; BARNES, A. C. \& GREGG, L. W. Injury Reduction Trends in Agricultural Aviation. Aerospace medicine, v. 35, n. May, p. 472-475, 1964.

CABLE, G. G. \& DOHERTY, S. Acute carbamate and organochlorine toxicity causing convulsions in an agricultural pilot: A case report. Aviation Space and Environmental Medicine, v. 70, n. 1, p. 68-72, 1999.

CANTOR, K. P. \& SILBERMAN, W. Mortality among aerial pesticide applicators and flight instructors: Follow-up from 1965-1988. American Journal of Industrial Medicine, v. 36, n. 2, p. 239-247, 1999.

http://dx.doi.org/10.1002/(SICI)1097-0274(199908)36:2<239::AID-AJIM3>3.0.CO;2-V

CENIPA. Acidentes na Aviação Civil 2001 a 2010. 2011.

CHATURVEDI, A. K. Aerospace toxicology overview: aerial application and cabin air quality. Rev Environ Contam Toxicol, v. 214, p. 15-40, 2011. http://dx.doi.org/10.1007/978-1-4614$\underline{0668-6 \quad 2}$

CHESTER, G. et al. Worker exposure to, and absorption of, cypermethrin during aerial application of an "ultra low volume" formulation to cotton. Archives of Environmental Contamination and Toxicology, v. 16, n. 1, p. 69-78, 1987.

http://dx.doi.org/10.1007/BF01055361

CHESTER, G. \& WARD, R. J. Occupational exposure and drift hazard during aerial application of Paraquat to cotton. Archives of Environmental Contamination and Toxicology, v. 13, n. 5, p. 551-563, 1984. http://dx.doi.org/10.1007/BF01056333

COHEN, B. et al. Sources of parathion exposures for Israeli aerial spray workers, 1977. Pesticides monitoring journal, v. 13, n. 3, p. 81-86, 1979.

DAUGHERTY, J. W.; LACEY, D. E. \& KORTY, P. Problems in aerial application .1. Some Biochemical effects of LINDANE and DIELDRIN on vertebrates. Aerospace medicine, v. 33, n. 10, p. 1171-1176, 1962.

DELLINGER, J. A. \& TAYLOR, H. L. Measuring the effects of neurotoxicants on flight simulator performance. Aviation Space and Environmental Medicine, v. 56, n. 3, p. 254257, 1985.

DRISKELL, W. J. et al. Methomyl in the blood of a pilot who crashed during aerial spraying. Journal of Analytical Toxicology, v. 15, n. 6, p. 339-340, 1991. http://dx.doi.org/10.1093/jat/15.6.339

Revista Produção Online, Florianópolis, SC, v.15, n. 4, p. 1195-1223, out./dez. 2015. 
FÆEREVIK, H. \& REINERTSEN, R. E. Effects of wearing aircrew protective clothing on physiological and cognitive responses under various ambient conditions. Ergonomics, v. 46, n. 8, p. 780-799, 2010. http://dx.doi.org/10.1080/0014013031000085644

FAR PART 137. Agricultural aircraft operations. 1965.

FITZPATRICK, D. T. An analysis of noise-induced hearing loss in army helicopter pilots. Aviation, Space, and Environmental Medicine, v. 59, n. 10, p. 937-941, 1988.

GERRY, A. C. et al. Low pilot exposure to Pyrethrin during ultra-low-volume (ULV) aerial inseticide application for control of adult mosquitoes. Journal of American Mosquito Control Association, v. 21, n. 3, p. 291-295, 2005. http://dx.doi.org/10.2987/8756971X(2005)21[291:LPETPD]2.0.CO;2

GORDON, M. \& HIRSCH, I. New issues in agricultural spraying in Israel. Aviation, space, and environmental medicine, v. 57, n. 6, p. 610-612, 1986.

GORDON, M. \& RICHTER, E. D. Hazards associated with aerial spraying of organophosphate insecticides in Israel. Rev Environ Health., v. 9, n. 4, p. 229-238, 1991. http://dx.doi.org/10.1515/REVEH.1991.9.4.229

GRIBETZ, B. et al. Heat stress exposure of aerial spray pilots. Aviation Space and Environmental Medicine, v. 51, n. 1, p. 56-60, 1980.

GUIMARÃES, A. Brasil possui segunda maior frota de aviação agrícola do mundo. Disponível em:http://www.cnt.org.br/Paginas/Agencia_Noticia.aspx?n=8480. Acesso em: 12 ago. 2011.

GUPTA, S. K. et al. Health risks in ultra-low-volume (ULV) aerial spray of malathion for mosquito control. J Environ Sci Health B., v. 15, n. 3, p. 287-294, 1980.

http://dx.doi.org/10.1080/03601238009372182

HALL, C. Agricultural pilot safety in Australia: A survey. Aviation Space and Environmental Medicine, v. 62, n. 3, p. 258-260, 1991.

HAVEN, H. DE. Development of crash-survival design in personal, executive and agricultural aircraft. Crash Injury Research. New York: Cornell University Medical College, 1953.

HRBA 137. Operações Aeroagrícolas. 1999.

JONES, S. M. et al. Occupational Asthma Symptoms and Respiratory Function Among Aerial Pesticide Applicators. American Journal of Industrial Medicine, v. 43, n. 4, p. 407-417, 2003. http://dx.doi.org/10.1002/ajim.10201 .

KÅSIN, J. I.; MANSFIELD, N. \& WAGSTAFF, A. Whole body vibration in helicopters: risk assessment in relation to low back pain. Aviation, Space, and Environmental Medicine, $v$. 82, n. 8, p. 790-6, 2011. http://dx.doi.org/10.3357/ASEM.2982.2011 .

KNARR, R. D. et al. Worker exposure during aerial application of a liquid and a granular formulation of ORDRAM selective herbicide to rice. Archives of Environmental

Revista Produção Online, Florianópolis, SC, v.15, n. 4, p. 1195-1223, out./dez. 2015. 
Contamination and Toxicology, v. 14, n. 5, p. 523-527, 1985.

http://dx.doi.org/10.1007/BF01055380

KNOPP, D. \& GLASS, S. Biological monitoring of 2,4-dichlorophenoxyacetic acid-exposed workers in agriculture and forestry. International Archives of Occupational and

Environmental Health, v. 63, n. 5, p. 329-333, 1991. http://dx.doi.org/10.1007/BF00381583

KÜPPER, T. E. et al. Noise exposure during ambulance flights and repatriation operations. International journal of occupational medicine and environmental health, v. 23, n. 4, p. 323-9, 2010. http://dx.doi.org/10.2478/v10001-010-0036-1

KURONEN, P. et al. Modelling the risk of noise-induced hearing loss among military pilots. International Journal of Audiology, v. 43, n. 2, p. 79-84, 2004.

http://dx.doi.org/10.1080/14992020400050013

LIBERATI, A. et al. The PRISMA statement for reporting systematic reviews and metaanalyses of studies that evaluate health care interventions: explanation and elaboration. PLoS medicine, v. 6, n. 7, p. 1-28, 2009. http://dx.doi.org/10.1371/journal.pmed.1000100

MINASI, L. B. et al. Cytogenetic damage in the buccal epithelium of Brazilian aviators occupationally exposed to agrochemicals. Genetics and molecular research, v. 10, n. 4, p. 3924-3929, 2011. http://dx.doi.org/10.1371/journal.pmed.100010010.4238/2011 .

MOHER, D. et al. Preferred reporting items for systematic reviews and meta-analyses: the PRISMA statement. PLoS medicine, v. 6, n. 7, p. 1-6, 2009.

http://dx.doi.org/10.1371/journal.pmed.1000097

MOHLER, S. R. Agriculture aviation medicine in the Soviet Union. Aviation, space, and environmental medicine, v. 51, n. 5, p. 515-517, 1980.

MORAES, A. De \& MONT'ALVÃO, C. Ergonomia: Conceitos e aplicação. 2a. edição ed. Rio de Janeiro: 2AB Editora Ltda, 2000.

NORTH, J. P. \& HIGHTOWER, R. D. Crop dusting injuries. American journal of surgery, v. 93, n. 4, p. 609-612, 1957. http://dx.doi.org/10.1016/0002-9610(57)90517-2

NTSB. Review of U.S. Civil Aviation Accidents: 2007 - 2009. Washington DC. 2011.

PARSONS, K. C. Environmental ergonomics: a review of principles, methods and models. Applied ergonomics, v. 31, n. 6, p. 581-94, 2000.

http://dx.doi.org/10.1016/S0003-6870(00)00044-2

PERRY, I. T. Some aspects of agricultural aviation in Australia. Chemistry \& Industry, n. 52, p. 1857-1858, 1969.

QUANTICK, H. R. Safety aspects of the aerial application of pesticides. Aeronaultical journal, v. 83, n. 821, p. 175-182, 1979.

QUANTICK, H. R. \& PERRY, I. C. Hazards of chemicals used in agricultural aviation: a review. Aviation Space and Environmental Medicine, v. 52, n. 10, p. 581-588, 1981.

Revista Produção Online, Florianópolis, SC, v.15, n. 4, p. 1195-1223, out./dez. 2015. 
REICH, G. A. \& BERNER, W. H. Aerial application accidents 1963 to 1966. An analysis of the principal factors. Archives of Environmental Health, v. 17, n. 5, p. 776-784, 1968. http://dx.doi.org/10.1080/00039896.1968.10665319

RICE, N. et al. Unplanned releases and injuries associated with aerial application of chemicals, 1995-2002. Journal of environmental health, v. 68, n. 4, p. 14-18, 2005.

RICHTER, E. et al. Exposures of aerial spray workers to parathion. Israel journal of medical sciences, v. 16, n. 2, p. 96-100, 1980.

RICHTER, E. et al. Death and injury in aerial spraying: Pre-crash, crash, and post crash prevention strategies. Aviation, space, and environmental medicine, n. 4, p. 53-56, 1981.

ROAN, C. C. et al. Spontaneous abortions, stillbirths, and birth defects in families of agricultural pilots. Archives of Environmental Health, v. 39, n. 1, p. 56-60, 1984. http://dx.doi.org/10.1080/00039896.1984.10545834

ROAN, C. C.; OLDS, K. L. \& MCILNAY, C. O. Pesticide Use and Pesticide Related Occupational Illness of Agricultural Pilots. World of Agricultural Aviation, v. 9, n. 3, p. 5660, 1982.

RYAN, G. A. \& DOUGHERTY, J. D. Epidemiology of aerial application accidents. Aerospace medicine, v. 40, n. 3, p. 304-309, 1969.

SAWINSKY, A. \& PASZTOR, G. Study of exposure to Reglone sprayed by aircraft. Zeitschrift fur die Gesamte Hygiene und Ihre Grenzgebiete, v. 23, n. 11, p. 845-846, 1977.

SIMPSON, G. R. Aerial spraying of organic phosphate pesticides. Lowered blood cholinesterase levels of aerial spray operators at Wee Waa. The Medical journal of Australia, v. 1, n. 15, p. 735-736, 1973.

SMITH, P. W. C.A.R.I. aeromedical symposium. Problems in aerial application. Aerospace medicine, v. 34, n. 6, p. 542-544, 1963.

SMITH, P. W.; STAVINOHA, W. B. \& RYAN, L. C. Cholinesterase inhibition in relation to fitness to fly. Aerospace Medicine, v. 39, n. 7, p. 754-758, 1968.

SMITH, S. D. Cockpit seat and pilot helmet vibration during flight operations on aircraft carriers. Aviation Space and Environmental Medicine, v. 75, n. 3, p. 247-254, 2004.

TESTUD, F. \& BOUGON, D. Intoxication sévère par un insecticide organophosphoré après accident de pulvérisation aérienne. Archives des Maladies Professionnelles et de l'Environnement, v. 70, n. 4, p. 465-470, 2009. http://dx.doi.org/10.1016/j.admp.2009.02.014

TOBIAS, J. V. Cockpit noise intensity - 3 Aerial application (Crop-dusting) aircraft. Journal of speech and hearing research, v. 11, n. 3, p. 611-615, 1968.

http://dx.doi.org/10.1044/jshr.1103.611

Revista Produção Online, Florianópolis, SC, v.15, n. 4, p. 1195-1223, out./dez. 2015. 
ULLMANN, L.; PHILLIPS, J. \& SACHSSE, K. Cholinesterase surveillance of aerial applicators and allied workers in the Democratic Republic of the Sudan. Arch Environ Contam Toxicol., v. 8, n. 6, p. 703-712, 1979. http://dx.doi.org/10.1007/BF01054871

VOOGT, A. J. DE; UITDEWILLIGEN, S. \& EREMENKO, N. Safety in high-risk helicopter operations: The role of additional crew in accident prevention. Safety Science, v. 47, n. 5, p. 717-721, 2009. http://dx.doi.org/10.1016/i.ssci.2008.09.009

WOOD, W. et al. Implication of organophosphate pesticide poisoning in the plane crash of a duster pilot. Aerospace medicine, v. 42, n. 10, p. 1111-1113, 1971.

WRIGHT, R. W. et al. How to write a Cochrane systematic review. Clinical orthopaedics and related research, v. 455, n. 455, p. 23-29, 2007.

http://dx.doi.org/10.1111/.1440-1797.2010.01380.x

YOSHIDA, K. et al. Measurements of surface contamination of spray equipment with pesticides after various methods of application. Journal of environmental science and health Part B Pesticides food contaminants and agricultural wastes, v. 25, n. 2, p. 169 183, 1990. http://dx.doi.org/10.1080/03601239009372682

YOSHIDA, K. et al. Characteristics of applicator exposure to synthetic pyrethroid in ULVhandheld and ULV-ULA spray applications. J Environ Sci Health B., v. 25, n. 2, p. 151-167, 1990. http://dx.doi.org/10.1080/03601239009372681

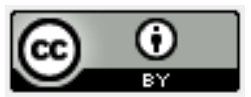

Artigo recebido em 09/05/2014 e aceito para publicação em 16/09/2015 DOI: http://dx.doi.org/ 10.14488/1676-1901.v15i4.1794 


\section{APÊNDICE}

Quadro 3 - Lista de artigos que foram referenciados na revisão de literatura.

\begin{tabular}{|c|c|}
\hline ESTUDOS & $\begin{array}{l}\text { MECANISMO E ALGORITMO } \\
\text { DE BUSCA }\end{array}$ \\
\hline VOOGT et al. (2009). & $\begin{array}{l}\text { Science Direct: (title-abs- } \\
\text { key((agricult* AND aviat*) } \\
\text { OR("aerial application"))). }\end{array}$ \\
\hline $\begin{array}{l}\text { (BILLINGS, 1963); (BRUGGINK et al., 1964); } \\
\text { (CHATURVEDI, 2011); (CHESTER \& WARD, 1984); } \\
\text { (CHESTER et al., 1987); (DAUGHERTY et al., 1962); } \\
\text { (DELLINGER \& TAYLOR, 1985); (GORDON \& HIRSCH, } \\
\text { 1986); (GORDON \& RICHTER, 1991); (GUPTA et al., } \\
\text { 1980); (HALL, 1991); (KNARR et al., 1985); (MINASI et } \\
\text { al., 2011); (MOHLER, 1980); (REICH \& BERNER, 1968); } \\
\text { (RICE et al., 2005); (RYAN \& DOUGHERTY, 1969); } \\
\text { (SMITH, 1963); (TOBIAS, 1968); (ULLMANN et al., } \\
\text { 1979); (YOSHIDA et al., 1990); }\end{array}$ & $\begin{array}{l}\text { NTSB (PubMed): (aviation[MeSH } \\
\text { Terms] AND agriculture[MeSH } \\
\text { Terms]). }\end{array}$ \\
\hline $\begin{array}{l}\text { (KNOPP \& GLASS, 1991); (PERRY, 1969); (QUANTICK } \\
\text { \& PERRY, 1981); (QUANTICK, 1979); }\end{array}$ & $\begin{array}{l}\text { ISI Web of Knowledge (Web of } \\
\text { Science): (TS=((agricult* AND } \\
\left.\text { aviat }^{\star}\right) \text { OR ("aerial application"))). }\end{array}$ \\
\hline $\begin{array}{l}\text { (COHEN et al., 1979); (DRISKELL et al., 1991); } \\
\text { (GRIBETZ et al., 1980); (HAVEN, 1953); (NORTH \& } \\
\text { HIGHTOWER, 1957); (RICHTER et al., 1980); } \\
\text { (RICHTER et al., 1981); (ROAN et al., 1982); } \\
\text { (SIMPSON, 1973); (WOOD et al., 1971); }\end{array}$ & $\begin{array}{l}\text { Estudos coletados manualmente } \\
\text { nas referências dos artigos } \\
\text { encontrados através dos } \\
\text { procedimentos de busca } \\
\text { descritos acima, seguindo os } \\
\text { mesmos critérios, mas aplicados } \\
\text { somente ao título informado. }\end{array}$ \\
\hline
\end{tabular}

